\title{
Recent Developments in Modeling Heteroepitaxy/ Heterogeneous Nucleation by Dynamical Density Functional Theory
}

\author{
FRIGYES PODMANICZKY, GYULA I. TÓTH, GYÖRGY TEGZE, \\ and LÁSZLÓ GRÁNÁSY
}

Crystallization of supersaturated liquids usually starts by epitaxial growth or by heterogeneous nucleation on foreign surfaces. Herein, we review recent advances made in modeling heteroepitaxy and heterogeneous nucleation on flat/modulated surfaces and nanoparticles within the framework of a simple dynamical density functional theory, known as the phase-field crystal model. It will be shown that the contact angle and the nucleation barrier are nonmonotonous functions of the lattice mismatch between the substrate and the crystalline phase. In continuous cooling studies for substrates with lattice mismatch, we recover qualitatively the Matthews-Blakeslee mechanism of stress release via the misfit dislocations. The simulations performed for particle-induced freezing will be confronted with recent analytical results, exploring thus the validity range of the latter. It will be demonstrated that time-dependent studies are essential, as investigations based on equilibrium properties often cannot identify the preferred nucleation pathways. Modeling of these phenomena is essential for designing materials on the basis of controlled nucleation and/or nano-patterning.

DOI: $10.1007 / \mathrm{s} 11661-015-2986-1$

(C) The Minerals, Metals \& Materials Society and ASM International 2015

\section{INTRODUCTION}

LIQUIDS cooled below their melting point are metastable with respect to crystallization, and exist in such a state until nucleation occurs; i.e., seeds of the crystalline phase form via thermal fluctuations. ${ }^{[1]}$ If these crystal-like fluctuations are larger than a critical size determined by the interplay of the driving force of freezing and the solid-liquid interface free energy, they grow further with a high probability, whereas the smaller ones tend to melt. The crystallites of the critical size are termed nuclei and their free energy of formation is the thermodynamic barrier of nucleation that the system needs to pass to reach the bulk crystalline state. Nucleation may be either homogeneous or heterogeneous. The homogeneous process is expected in an idealized pure undercooled liquid (which is well approximated by colloidal systems), where the internal fluctuations of the liquid enable the system in passing of the thermodynamic barrier toward crystallization, whereas heterogeneous nucleation takes place in "impure" liquids, where

FRIGYES PODMANICZKY, Ph.D. Student, and GYÖRGY TEGZE, Senior Scientist, are with the Wigner Research Centre for Physics, P.O.Box 49, Budapest 1525, Hungary. GYULA I. TÓTH, Post-Doctoral Research Fellow, is with the Wigner Research Centre for Physics, and also with the Department of Physics and Technology, University of Bergen, Allégaten 55, 5007 Bergen, Norway. LÁSZLO GRÁNÁSY, Professor, Scientific Advisor, is with the Wigner Research Centre for Physics, and also with the BCAST, Brunel University, Uxbridge, Middlesex UB8 3PH, U.K. Contact e-mail: granasy.laszlo@wigner.mta.hu

Manuscript submitted September 18, 2014. foreign surfaces (container walls or the perimeter of foreign particles) assist nucleation by reducing the free energy barrier associated with crystal nucleation. This happens, when the ordering of the liquid layers adjacent to the foreign wall helps the formation of the crystalline phase. This is, however, not necessarily so: a foreign wall may have a structure that is incompatible with that of the crystal, which may even hinder nucleation. Besides the essential role played in materials science, heterogeneous nucleation attracts a growing attention recently due to the emerging technological interest, for example, in micro- and nanopatterning techniques, ${ }^{[2]}$ and controlling the related nanoscale processes, such as crystallization on patterned surfaces, the formation of quantum dots, ${ }^{[3-6]}$ the controlling of the properties of partly crystalline glass ceramics, ${ }^{[1]}$ phase selection in alloys, ${ }^{[7]}$ and nucleation of metals on graphene. ${ }^{[8]}$ Particulate additives are often used as nucleating agents to control the grain size of the solidified matter. ${ }^{[1]}$ A similar mechanism appears to control the undercoolability of living organisms. ${ }^{[1]}$ Despite its practical importance, and recent investigations in the field, ${ }^{[9-18]}$ which include atomistic simulations, ${ }^{[9-14]}$ phase-field, ${ }^{[15,16]}$ and density functional studies, ${ }^{[17,18]}$ further work is needed to clarify the interaction between the foreign surfaces/particles (henceforth termed "substrate") and the solidifying melt.

The events at such surfaces are often categorized considering the relative interfacial free energies of the crystal-liquid $\left(\gamma_{\mathrm{SL}}\right)$, crystal-wall $\left(\gamma_{\mathrm{SW}}\right)$, and liquid-wall $\left(\gamma_{\mathrm{LW}}\right)$ interfaces. Introducing the quantity, $\Delta \gamma=\gamma_{\mathrm{SL}}+$ $\gamma_{\mathrm{SW}}-\gamma_{\mathrm{LW}}$, the possible scenarios can be divided into three categories: 
(i) Layer-by-layer growth (or van der Meerwe mechanism) that occurs when $\Delta \gamma<0$;

(ii) Layer-by-layer growth up to a critical thickness followed by island formation (Stranski-Krastanov mechanism), appearing for $\Delta \gamma \approx 0$; and

(iii) Island formation (Volmer-Weber mechanism, or heterogeneous nucleation), which happens if $\Delta \gamma>$ 0 .

The efficiency of a foreign wall in promoting crystallization is influenced by such microscopic properties as crystal structure, lattice mismatch, surface roughness, adsorption, etc., which require atomistic modeling. Atomistic simulations have been used extensively to address the interaction between foreign walls and crystallizing fluids. ${ }^{[9-14]}$

The foreign surfaces are known to establish structural ordering in the adjacent liquid layers, ${ }^{[13,14]}$ a phenomenon that naturally influences the adsorption of crystalline molecule layers at the surface of the substrate, which is of primary importance from the viewpoint of the free-growth limited model of particle-induced freezing proposed by Greer and coworkers. ${ }^{[1,19-21]}$ The latter model assumes cylindrical particles, whose circular faces (of radius $R$ ) are ideally wet by the crystal. It follows then that the particles remain dormant during cooling so far as the radius of the homogeneous nuclei is larger than $R$. Beyond this limit, however, free growth sets in. (The critical undercooling at which free growth starts is expressible as $\Delta T_{\mathrm{c}} \approx 2 \gamma_{\mathrm{SL}} /\left(\Delta s_{\mathrm{f}} R\right)$, where $\Delta s_{\mathrm{f}}$ is the volumetric melting entropy.) This particle-initiated mechanism of freezing has been recovered recently using a conventional phase-field model that relies on a coarsegrained structural order parameter. ${ }^{[15]}$ It has also been shown that for nanoparticles, the effect of fluctuations can be essential: with the fluctuation dissipation noise, the critical undercooling has been roughly halved, whereas without fluctuations results from the analytic theory have been recovered. ${ }^{[15]}$ The same mechanism has been investigated recently by atomistic simulations based on a simple dynamical density functional theory (DDFT), known as the phase-field crystal (PFC) model. ${ }^{[2,23]}$ Such simulations have recently been used for determining the validity range of the analytic model. ${ }^{[24,25]}$ An extension of the model to the case, when wetting of the surface of the particles is non-ideal due to lattice mismatch, has been discussed by Fan. ${ }^{[26,27]}$ Further atomistic scale studies that address heteroepitaxy on substrates/particles of various crystal structures are desirable in this respect.

It is also worth noting that according to recent investigations even homogeneous nucleation often appears to be a two-stage process, in which the crystalline phase appears on amorphous "particles" formed earlier; i.e., crystallization is assisted by an amorphous precursor state. This process may be regarded as a specific heterogeneous nucleation phenomenon. A similar twostep process is observed in experiments on solutions of globular proteins, where phase separation in the liquid state helps the formation of crystal nuclei, especially at the critical point, leading to the formation of composite nuclei made of a crystallite surrounded by a dense liquid. ${ }^{[28,29]}$ This behavior has also been reproduced in atomistic simulations ${ }^{[30]}$ and density functional/ phase-field computations. ${ }^{[31,32]}$ Brownian dynamics simulations indicate the formation of medium range crystal-like order in the supercooled liquid preceding crystallization. ${ }^{[33]}$ Further systems that show analogous behavior are colloids, ${ }^{[34,35]}$ simple liquids (LennardJones $^{[36]}$ or hard-sphere ${ }^{[37]}$, or systems with DeryaguinLandau-Verwey-Overbeek-type potential, ${ }^{[38]}$ where the appearance of the crystalline phase is preceded by an amorphous/dense liquid precursor. It appears that precursor-assisted "homogeneous" crystal nucleation is a general phenomenon.

The atomic scale aspects of homogeneous ${ }^{[38]}$ and heterogeneous ${ }^{[24,25]}$ crystal nucleation have recently been studied by the PFC model. In this model, several crystalline phases (bcc, hcp, and fcc) compete with an amorphous solid during freezing. ${ }^{[23]}$ The PFC model works on a diffusive time scale, and can be regarded complementary to molecular dynamics. ${ }^{[23]}$ Dynamic PFC simulations indicate that beyond a critical supersaturation the density and structural changes decouple, leading to amorphous precursor-mediated freezing. ${ }^{[23,38]}$ The effect of lattice mismatch on the heterogeneous process $^{[23-25]}$ has also been explored.

Herein, we concentrate on recent advances made by PFC modeling of crystallization in the presence of foreign surfaces. Our paper is structured as follows: In Section II, we recapitulate the main features of the PFC model. Section III contains the parameter values and conditions used in the simulations, whereas Section IV is devoted to a study of the three essential mechanisms of solidification on solid surfaces: layer-by-layer growth, layer-by-layer growth up to a critical thickness followed by island formation, and island formation, together with exploring of the microscopic aspects of particle-induced freezing. Finally, a few concluding remarks are offered in Section V.

\section{PFC MODELS}

The main features of the PFC models have recently been reviewed in Reference 23. These models can be considered as simple classical dynamic density functional theories, in which the local state of matter is characterized by a particle density field $\psi(\mathbf{r}, t)$ coarse grained in time that depends on position, r, and time, $t$. Owing to time averaging, the particle density is homogeneous in the liquid, whereas density peaks appear in the crystal at the particle positions. An overdamped conservative dynamics is assumed yielding a diffusive equation of motion (EOM), whereas the equilibrium properties, including the interface free energy and the phase diagram are evaluated using the Euler-Lagrange equation (ELE). ${ }^{[23]}$

\section{A. The Free Energy}

Herein, we rely on the following form of the dimensionless free energy 


$$
\Delta F=\int d \mathbf{r}\left\{\frac{\psi}{2}\left[-\varepsilon+\left(1+\nabla^{2}\right)^{2}\right] \psi+\frac{\psi^{4}}{4}+V \psi\right\},
$$

where $\Delta F$ is measured relative to the free energy of a homogeneous reference liquid, $\psi$ is a reduced singlet (particle) density, whereas $\mathbf{r}$ is the dimensionless spatial position, while $\varepsilon>0$ is the distance from a critical point located at $\psi=0, \varepsilon=0$. The reduced temperature $\varepsilon$ is expressible in terms of the compressibility of the liquid, the bulk modulus of the solid, and the lattice constant. It has been shown that Eq. [1] for the free energy with $V=$ $0(=$ the free energy of the original single component PFC model of Elder et al. ${ }^{[22]}$ ) can be deduced from a perturbative density functional approach via simplifications including the expansion of the two-particle direct correlation function in Fourier space. ${ }^{[39,40]}$ In dimensionless form, the free energy reduces to a Brazovskii/ Swift-Hohenberg form. ${ }^{[22,23]}$ The applied approximations lead to a preferred wavelength for the particle density. Accordingly, periodic density distributions that are consistent with this wavelength correspond to local minima of the free energy. Thus, elasticity and crystal anisotropies are automatically incorporated. This model has been applied successfully for a broad range of phenomena, ${ }^{23]}$ incorporating polymorphism, dendritic/ eutectic growth, glass formation, grain boundary dynamics, melting at grain boundaries, etc. The phase diagram of the original PFC model $(V=0)$ is shown in Figure 1.

To incorporate a crystalline substrate into the model, the original PFC expression for free energy density has been supplemented with a periodic potential term
$V(\mathbf{r}) \psi^{[23,24]}$ (See the third term of the integrand on the RHS of Eq. [1].) Here $V(\mathbf{r})=\left[V_{\mathrm{s}, 0}-V_{\mathrm{s}, 1} S\left(a_{\mathrm{s}}, \mathbf{r}\right)\right] h(\mathbf{r})$, while $V_{\mathrm{s}, 0}$ and $V_{\mathrm{s}, 1}$ are constants, of which the first controls the crystal adsorption, whereas $V_{\mathrm{s}, 1}$ is the amplitude of the periodic part. The function $S\left(a_{\mathrm{s}}, \mathbf{r}\right)$ is an approximate analytical solution that ensures a periodic structure for the substrate, ${ }^{[23,24]} a_{\mathrm{s}}$ is the lattice constant of the substrate, whereas $h(\mathbf{r}) \in[0,1]$ is an envelope function that defines the domain filled by the substrate. ${ }^{[23,24]}$

After having specified the free energy functional, nucleation can be addressed in two ways: (i) either via solving the Euler-Lagrange equation (ELE) under the appropriate boundary conditions obtaining thus the critical fluctuation (nucleus); or (ii) by solving the equation of motion (EOM) with noise representing thermal fluctuations one simulates nucleation. Route (i) is fully consistent with the free energy functional. However, owing to the noise applied in the case of route (ii), the free energy of the phases change together with the phase diagram and the interfacial properties. Decreasing the noise amplitude, results from route (ii) converge to those from route (i). The full richness of the nucleation pathways can often be revealed by applying both routes simultaneously.

\section{B. Equation of Motion}

The time evolution of the system is described by the EOM. We note that the particle density is a conserved field. Accordingly, overdamped conservative (diffusive) dynamics was adopted in the PFC model, ${ }^{[22]}$ which is realized by the dimensionless equation
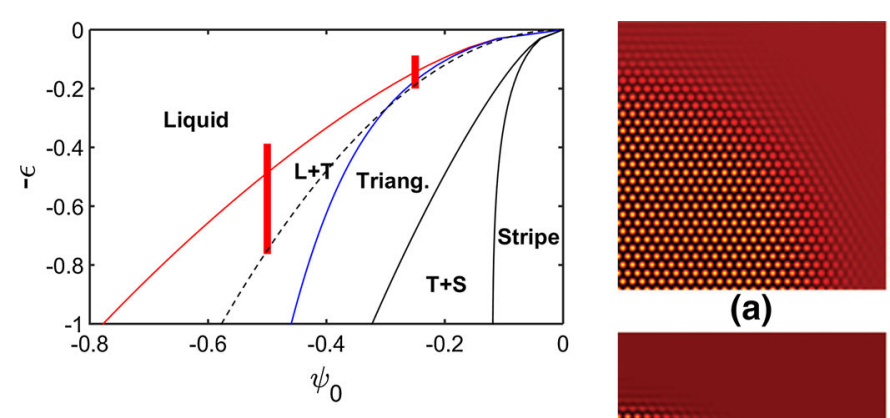

(a)

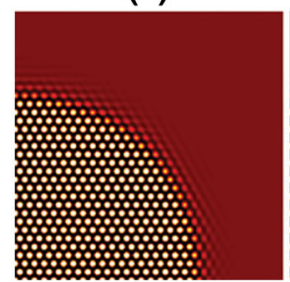

(e)

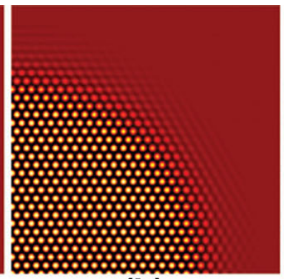

(b)

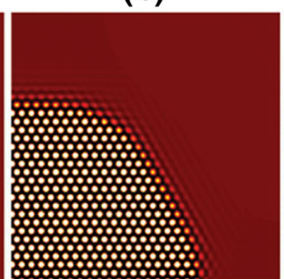

(f)

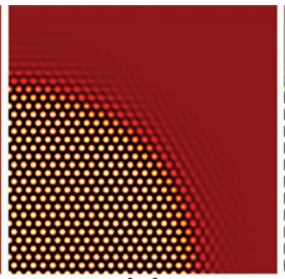

(c)

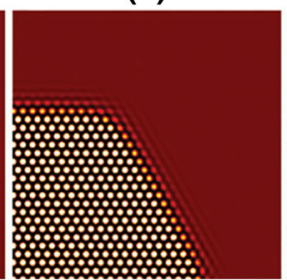

(g)

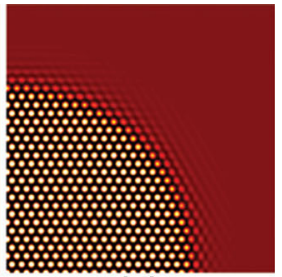

(d)

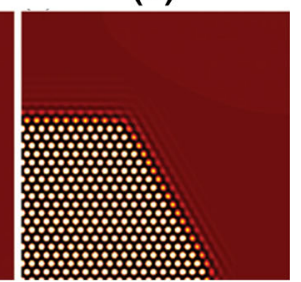

(h)

Fig. $1-$ Relevant properties of the PFC model $(V=0)$ in 2D. Left: Phase diagram in the negative reduced temperature $(-\varepsilon)$ and reduced density $\left(\psi_{0}\right)$ plane. The thin solid lines denote the borders of the stability domains: liquidus -1 st from left, solidus -2 nd from left. The dashed line is the linear stability limit of the liquid, to the right of which it is unstable with respect to solidification. " $\mathrm{L}+\mathrm{T}$ " stands for the coexistence region of the liquid and triangular phases; "Triang." denotes the triangular crystal structure, "Stripe" stands for a striped or lamellar phase, irrelevant from the viewpoint of this study, but present at large supersaturations, whereas " $T+S$ " denotes the coexistence domain of the triangular and striped phases. Supersaturation is defined here as $\mathrm{S}=\left(\psi_{0}-\psi_{0, \mathrm{~L}}\right) /\left(\psi_{0, \mathrm{~S}}-\psi_{0, \mathrm{~L}}\right)$, where $\psi_{0, \mathrm{~S}}$ and $\psi_{0, \mathrm{~L}}$ are the reduced densities at the solidus and liquidus lines at the given reduced temperature, $\varepsilon$. The reduced undercooling corresponding to a reduced density $\psi_{0}$, in turn, is defined as $\Delta \varepsilon=\varepsilon\left(\psi_{0}\right)-$ $\varepsilon_{\mathrm{L}}\left(\psi_{0}\right)$, where the latter term stands for the liquidus line. The thick vertical bars display the paths for the cooling processes described in the text. Right: Wulff shapes reflecting the anisotropy of the crystal-liquid interface are shown as a function of the reduced temperature, $\varepsilon$ for a crystalline fraction of $X=0.3$, in the absence of noise. ( $a$ through $h$ ): $\varepsilon=0.05,0.10,0.15,0.20,0.25,0.30,0.325$, and 0.35 . The interface thickness decreases while the anisotropy increases with an increasing distance from the critical point. In the numerical solution of the EOM, a $1024 \times 1024$ rectangular grid was used (the upper right quarter is shown). Equilibration was performed for a period of $10^{6}$ dimensionless time steps. 


$$
\frac{\partial \psi}{\partial t}=\nabla^{2} \frac{\delta \Delta F}{\delta \psi}+\zeta
$$

The thermal fluctuations are represented by colored Gaussian noise $\zeta$ of correlator $\left\langle\zeta(\mathbf{r}, t) \zeta\left(\mathbf{r}^{\prime}, t^{\prime}\right)\right\rangle=$ $-A_{\text {noise }}^{2} \nabla^{2} g\left(\left|\mathbf{r}-\mathbf{r}^{\prime}\right|, \sigma\right) \delta\left(t-t^{\prime}\right)$. Here, $A_{\text {noise }}$ is the noise strength and high frequency cutoff is realized by the function $g\left(\left|\mathbf{r}-\mathbf{r}^{\prime}\right|, \sigma\right)$ for wavelengths smaller than the interparticle spacing, $\sigma{ }^{[23-25]}$ We note that $A_{\text {noise }}$ is a different combination of the physical parameters than $\varepsilon$, thus they are independent of each other. This EOM has been deduced from the DDFT of colloids, after making simplifications. ${ }^{[23,40]}$ The PFC models relying on this type of EOM are appropriate for modeling crystalline particle aggregation in colloidal systems.

We note here that in the classical DDFT-type models, nucleation does not occur in a homogeneous liquid unless Langevin noise is added to the EOM, which represents the thermal fluctuations. Although this procedure mimics nucleation, it is not free from conceptual difficulties, as discussed in the literature ${ }^{[41-43]}$ Assuming that the particle density is an ensemble averaged quantity, it follows that the free energy contains all the possible fluctuations. Then adding noise to the equation of motion should mean that part of the fluctuations are counted doubly. ${ }^{[1,42]}$ Assuming, however, that the number density is a time averaged quantity, it appears justifiable to add noise to the EOM to model the thermal fluctuations. ${ }^{[43]}$ Then, both crystal nucleation and capillary waves at the crystalliquid interface appear automatically. In the majority of the recent dynamic PFC studies of crystal nucleation on the atomistic scale, ${ }^{[23-25,38]}$ a colored flux noise is used in the EOM obtained by filtering out the unphysical short wavelengths.

\section{Euler-Lagrange Equation}

The equilibrium features of the PFC system can be deduced from the free energy functional by solving the Euler-Lagrange equation (ELE):

$$
\frac{\delta \Delta F}{\delta \psi}=\left.\frac{\delta \Delta F}{\delta \psi}\right|_{\psi_{0}} .
$$

Here, $\psi_{0}$ is the reduced particle density of the reference liquid, while the term on the RHS of Eq. [3] $\delta \Delta F /\left.\delta \psi\right|_{\psi=\psi_{0}}=\mu_{0}$ is the chemical potential of the reference liquid. Periodic boundary condition is prescribed at the borders of the simulation box. Inserting the free energy functional from Eq. [1] into Eq. [3], one obtains

$$
\left[-\varepsilon+\left(1+\nabla^{2}\right)^{2}\right]\left(\psi-\psi_{0}\right)=-\left(\psi^{3}-\psi_{0}^{3}\right)-V,
$$

for the ELE, which represents a 4th order boundary value problem when combined the boundary conditions. The ELE method ${ }^{[44]}$ has been widely used to obtain equilibrium properties of the PFC system ${ }^{[23,44]}$ including the phase diagram, the solid-liquid interface free energy, the density difference at the solid-liquid interface, and the nucleation barrier for bcc and fcc structures in $3 \mathrm{D}$.

\section{Numerical Methods}

Solutions of the EOM and ELE have been obtained numerically. The ELE has been solved by using a pseudo-spectral successive approximation scheme based on the operator-splitting method. In the case of the EOM, a similar approach relying on a semi-implicit spectral scheme ${ }^{[45]}$ has been used, which proved highly efficient for solving the PFC-type EOMs. The computations were performed using various types of GPU (Graphics Processing Unit) cards.

\section{SIMULATION CONDITIONS}

\section{A. Spatial and Time Steps}

In solving the EOM, the dimensionless spatial and time steps were chosen as $\Delta x=(4 \pi / \sqrt{ } 3) / 20$, and $\Delta t=$ 0.1 , respectively. The amplitude of the noise added was varied in a broad range $\left(10^{-7}<A_{\text {noise }}<1\right)$, however, if not stated otherwise $A_{\text {noise }}=10^{-4}$ has been applied.

\section{B. Tuning the Anisotropy}

In the PFC model, the thickness and anisotropy of the solid-liquid interface vary with the reduced temperature $\varepsilon$ between essentially isotropic and broad interface (near the critical point) and strongly anisotropic and sharp interface (far from the critical point). Accordingly, the equilibrium shapes vary between circle and hexagon (see Figure 1). ${ }^{[23,44,46,47]}$ As a result, by the choice of $\varepsilon$ one may select qualitative features characteristic to different classes of materials. In the isothermal simulations, two typical reduced temperature values were used: (a) at $\varepsilon=$ 0.25 , the model provides a small anisotropy and a broad solid-liquid interface, characteristic to metals, and (b) at $\varepsilon=0.5$ an interface sharp on the atomic scale and strongly faceted develops, mimicking covalent systems.

In the constant cooling rate simulations, we have assumed initial reduced densities of $\psi_{0}=-0.25$ and $\psi_{0}$ $=-0.5$. For these, cooling started from above the liquidus line and ended at the linear stability limit of the liquid phase $\left(\varepsilon_{\mathrm{c}}=3 \psi_{0}^{2}\right)$, corresponding to $\varepsilon \in[0.1$, $0.1875]$ and $\varepsilon \in[0.4,0.75]$, respectively, whereas the cooling time has been chosen as $3 \times 10^{5} \Delta t$. Figure 1 displays these cooling paths in the phase diagram by heavy vertical bars. Along these cooling paths, the anisotropy continuously varies to some extent, and however, remains typically low (metal-like) for the first case, and high (covalent-like) for the second.

\section{Modeling Fluctuations by Noise}

The PFC model contains only a reduced temperature, measured with respect to a hypothetical critical point. An approximate temperature scale can be obtained if the model is fitted to properties of a real material. This temperature scale, however, varies from matter to 
matter, and a general handling is not available. Therefore, following other work in the literature, in our simulations the noise amplitude, which should in principle be temperature dependent, has been treated as a model parameter and is kept constant. This uncertainty associated with the noise affects only the simulations made by the EOM; the results from solving the ELE (Figures 1, 7, 8, 11, and 12) are free from it. For the simulations performed with EOM (Figures 2, 3, 4, 5, 6, 9,10 , and 13), we tested the effect of noise strength, and found that the qualitative behavior remains similar in a broad noise amplitude range.

We note that adding noise to a mean field type model like the PFC has conceptual difficulties (as discussed in the literature, see e.g., Reference 23), which emerges from the fact that a part of the fluctuations is already integrated into the free energy, yet another part, the long wavelengths are obviously missing, as there are no capillary waves and no nucleation without adding noise to the equation of motion.

\section{Modeling a Crystalline Substrate}

In the simulations, a substrate of square-lattice was used, for which the single mode approximant has the following form:

$$
S(q, \mathbf{r})=\cos (q x)+\cos (q y),
$$

where $q$ is varied, and $x$ and $y$ are the components of $\mathbf{r}$.

The envelope function, $h(\mathbf{r})$, incorporates a $\tanh [n /$ $\left.\left(w \sigma_{0}\right)\right]$ smoothing at the surface of the substrate. Here $n$ is a spatial coordinate perpendicular to the surface, $w$ is the thickness parameter, whereas $\sigma_{0}$ is the interparticle distance in the crystal. In most of the studies, a relatively sharp surface $(w=1 / 8)$ was chosen for the substrate. The dependence of the

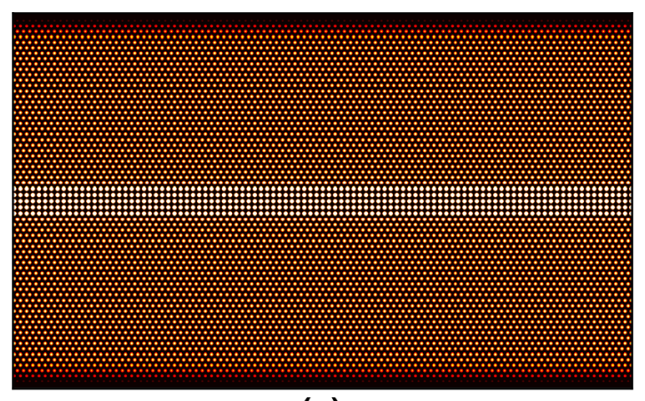

(a)

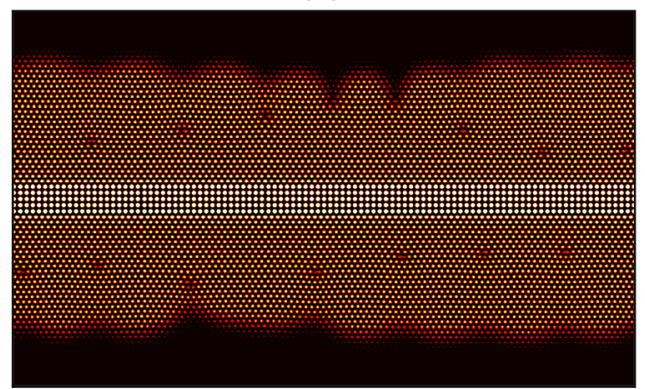

(b)

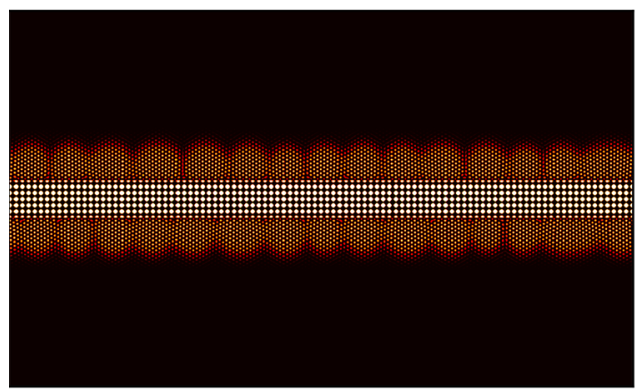

(c)

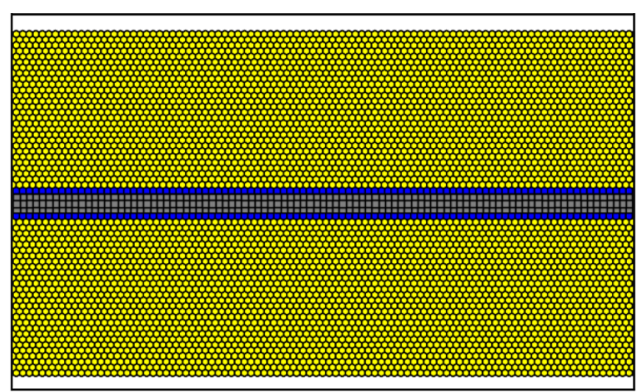

(d)

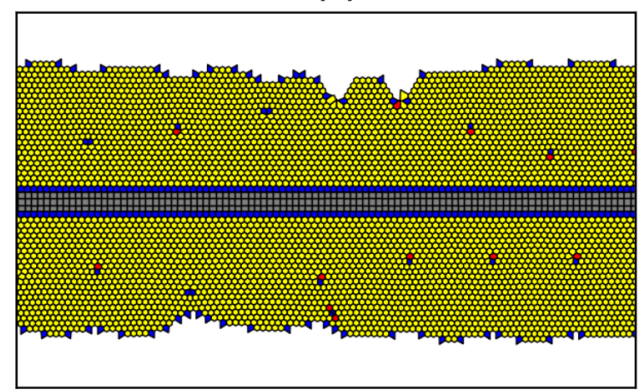

(e)

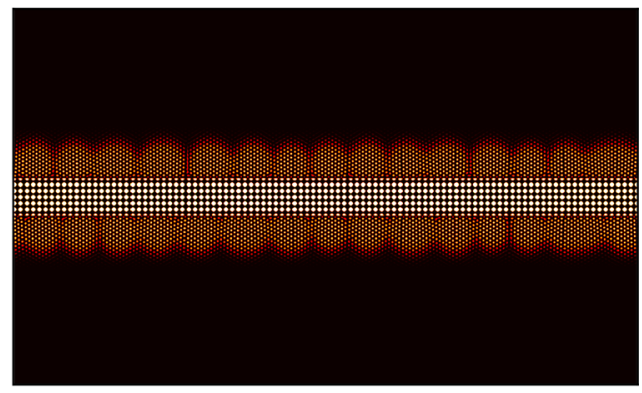

(f)

Fig. 2-Heteroepitaxy in continuous cooling as predicted by the PFC model in the case of low anisotropy of the free energy of the crystal-liquid interface. (Here $\psi_{0}=-0.25$, while the reduced temperature $\varepsilon$ increases linearly in time from 0.1 to 0.1875 (corresponding to cooling from above the melting point, and essentially isotropic solid-liquid interface free energy) in $3 \times 10^{5}$ dimensionless time steps): $(a$ through $c)$ and $(f)$ Particle density maps. $(d, e)$ Voronoi polyhedral analysis. (a, d) Layer-by-layer growth (van der Meerwe mechanism); (b, e) layer-by-layer growth followed by island formation (Stranski-Krastanov mechanism); and (c, $f$ ) island formation (Volmer-Weber mechanism/heterogeneous nucleation). In panels (d) and (e) the colors correspond to different numbers of the neighbors: gray, blue, yellow, and red stand for 4, 5, 6, and 7 neighbors, respectively. From top to bottom $\sigma / \sigma_{0}=1.0,1.07$, and 1.60 . 
results on $w$ is weak in the range of $w=[1 / 8,1 / 2]$. In the simulations for heteroepitaxy, the width of the simulation window $L_{x}$ (taken equal to the length of the substrate) has been chosen so that it is commensurable with both the substrate and the unstrained crystal.

Remarkably, if one starts from a uniform initial density in the presence of a substrate, the respective potential wells need to be filled by matter. Owing to the diffusive dynamics prescribed by the EOM, the filling happens via diffusion from the regions adjacent to the substrate, leaving so a slowly disappearing depletion zone around the substrate, from which matter moved into the substrate. In this depleted zone, the supersaturation is low; therefore, crystallization is suppressed. To avoid this problem, we use the ELE to create an initial density distribution, in which the filled substrate is in equilibrium with the liquid.

\section{RESULTS AND DISCUSSION}

\section{A. Flat Substrate}

First, the results of constant cooling rate simulation for the low anisotropy (metal-like) case $(\varepsilon \in[0.1$, $0.1875])$ are presented. The misfit $f$ has been varied in the range of $-0.12<f<0.11$ [here $f=\left(\sigma_{0}-\sigma\right) / \sigma$, where $\sigma$ is the interparticle distance in the substrate]. Depending on the magnitude of misfit, we have observed all the three modes of heteroepitaxy (Figure 2). Besides showing the particle density maps (Figures 2(a) through (c)), the results of structural analysis in terms of Voronoipolyhedra are also shown (Figures 2(d) and (e)). In the latter case, particles with different numbers of neighbors are colored differently: gray, blue, yellow, and red colors stand for 4, 5, 6, and 7 neighbors, respectively. The dislocations consist of a pair of disclinations (i.e., 5 and 7 coordinated particles, represented by a blue-red pair).

For $f \rightarrow 0$, the critical thickness $h_{\mathrm{c}} \rightarrow \infty$, as expected from the Matthews-Blakeslee theory. ${ }^{[48]}$ Accordingly, in a neighborhood of $f=0$, where the vertical size of the simulation window is less than $h_{\mathrm{c}} / 2$, we observe a flat growth front (layer-by-layer growth albeit with a diffuse interface, see Figures 2(a) and (d)). For larger $f$, however, growth with a flat front occurs first, which becomes undulated due to the Asaro-Tiller-Grinfeld instability $^{[49]}$ (Figures 2(b) and (e)). As the respective cusps become pronounced, misfit dislocations nucleate at the bottom of the cusps that release the stress/strain, and march toward the substrate-crystal interface, where they form a quasi-periodic arrangement. The remaining solid is usually not yet fully stress free, so further misfit dislocations are generated. When the misfit is sufficiently high, island formation occurs (Figures 2(c) and (f)).

If the Matthews-Blakeslee scaling is obeyed, plotting $\left[1+\ln \left(h_{\mathrm{c}} / b\right)\right] /\left(h_{\mathrm{c}} / b\right)$ with respect to $f$ should result in a straight line crossing the origin. ${ }^{[48]}$ (Here $b$ is the length of the Burgers vector, which is $\sigma_{0}$ in our case.) Indeed, the Matthews-Blakeslee plot for this nearly isotropic system is close to linear (Figure 3 ). These results seem to be in a reasonable agreement with the findings of Elder and Grant, ${ }^{[50]}$ who used a substrate of triangular structure, realized in a different way in the PFC model.

Next, we present the results obtained in continuous cooling runs with high anisotropy ( $\varepsilon$ changes linearly with time between 0.4 and 0.75 ). Here, the misfit $f$ has been changed in the range of $-0.22<f<-0.16$. The three modes of heteroepitaxy has been again recovered (Figure 4). A behavior consistent with the divergence of $h_{\mathrm{c}}$ toward zero misfit has been observed again (Figure 5(a)), however, for larger misfits. Remarkably, here the amplitude of the Asaro-Tiller-Grinfeld modulation of the interface is considerably smaller than in the case of the low anisotropies. It is also remarkable that although the Matthews-Blakeslee plot is close to linear, it does not extrapolate to the origin (Figure 5(b)). To reach the origin, a considerable curvature is needed. This result requires further investigations. The origin of the deviation from the Matthews-Blakeslee scaling is presumably the non-linear elasticity prevailing far from the critical point. Work is underway to clarify this point.

Apparently, the addition of noise to the EOM influences the onset of the Asaro-Tiller-Grinfeld instability, though the qualitative behavior remains the same in a broad range of $A_{\text {noise }}$. The results are summarized in Figure 6. Here, the critical thickness has been determined for large anisotropy $\left(\psi_{0}=-0.5\right.$, while $\varepsilon \in[0.4$, 0.75]) during continuous cooling, while varying the amplitude of the dimensionless flux noise between $A_{\text {noise }}$ $=10^{-7}$ and $A_{\text {noise }}=1$. Remarkably, in most of the investigated regime, the critical thickness decreases exponentially with increasing amplitude of the flux noise, whereas above $A_{\text {noise }}=10^{-3}$ the critical thickness drops suddenly to a monolayer (Figure 6). It is worth noting that in the latter regime the defects appear directly at the substrate-crystal interface from the very beginning. Further work is needed to identify the details of this sharp transition in the mechanism of defect formation.

\section{Island formation/heterogeneous nucleation}

Previous work investigated heterogeneous nucleation on a flat substrate of square-lattice structure while varying its lattice constant. ${ }^{44]}$ It has been observed that owing to the atomistic nature of the theory the free energy surface has many local minima allowing the Euler-Lagrange equation to map out the nucleation barrier (see Figure 7). The dominant relative orientations observed in dynamic simulations were faces (01-1) or (11-2) parallel to the wall. The dependence of the contact angle $\theta$ on the lattice mismatch has been evaluated for weak anisotropy. ${ }^{[2]}$ The definition adopted for $\theta$ in Reference 24 is the angle between the linear and circular parts of the closed contour line corresponding to $\left(\psi_{\mathrm{L}}+\psi_{\mathrm{S}}\right) / 2$ in the coarse-grained particle density (see Figures $8(\mathrm{a})$ and (b)). (Here $\psi_{\mathrm{L}}$ and $\psi_{\mathrm{S}}$ are the particle densities in the bulk solid and liquid phases.) A nonmonotonic relationship is observed between the contact angle and the reduced lattice constant $\left(\sigma / \sigma_{0}\right)$ (Figure 8(c)). In contrast, for strong anisotropy that yields faceted interfaces, the contact angle is determined by the crystal structure and orientation: $\theta=60 \mathrm{deg}$ 


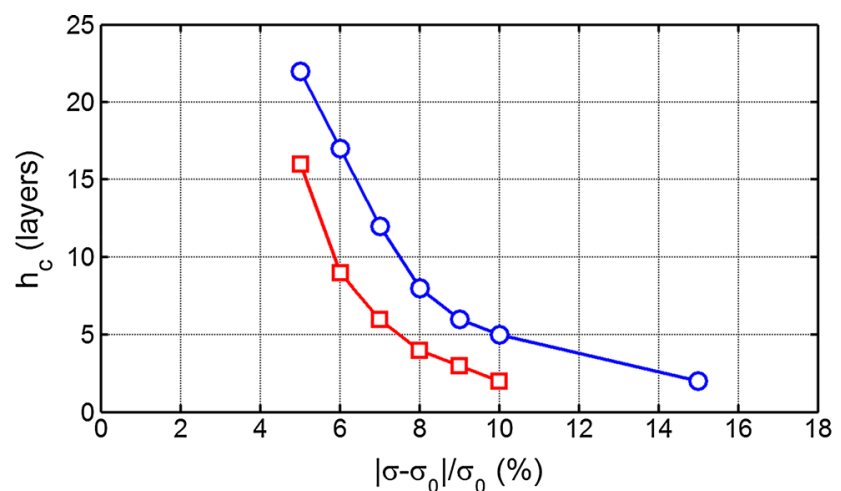

(a)

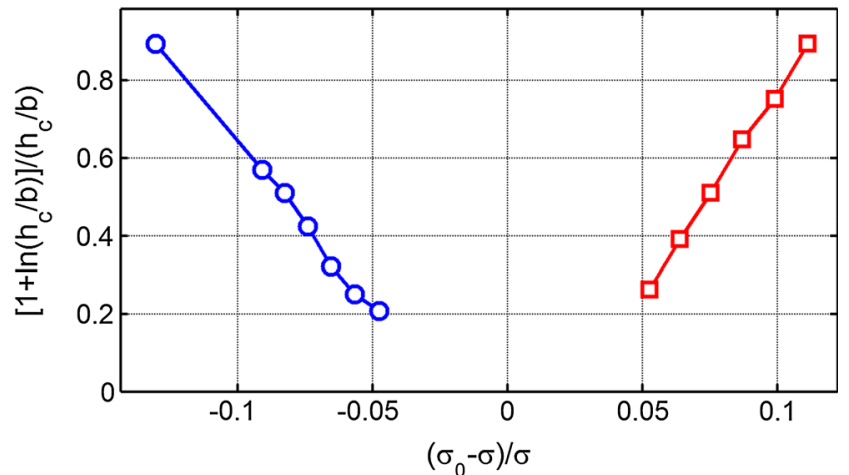

(b)

Fig. 3-Stress release via misfit dislocations in metal-like (low anisotropy) systems: (a) Critical thickness of layer-by-layer growth vs lattice mismatch for positive (squares) and negative (circles) mismatch. (b) The Matthews-Blakeslee plot.

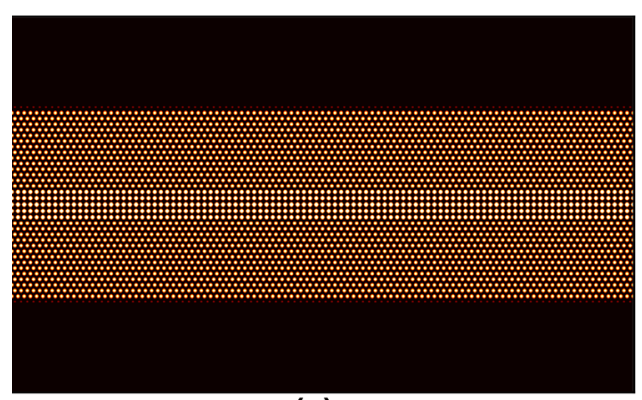

(a)

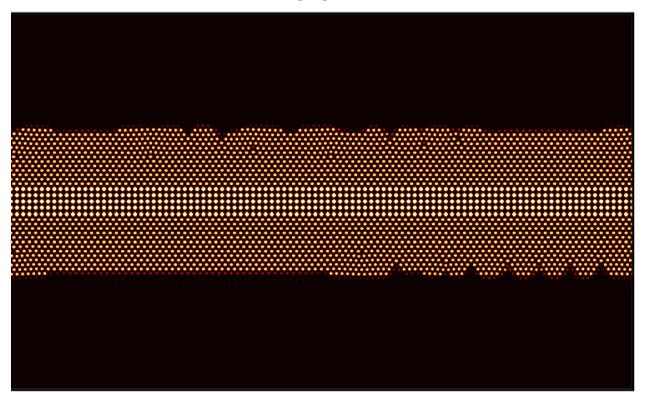

(b)

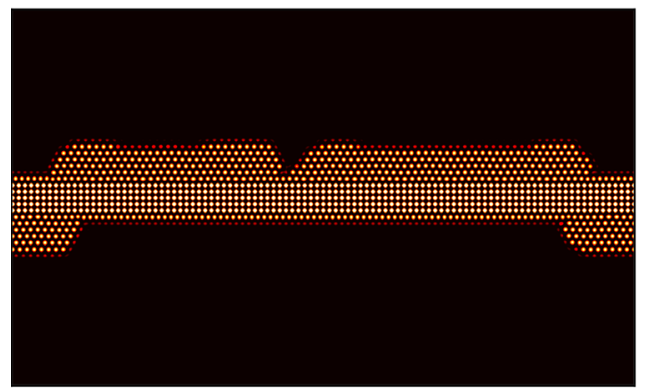

(c)

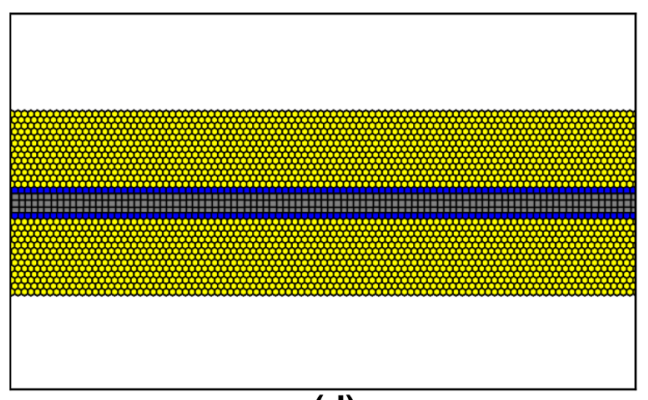

(d)

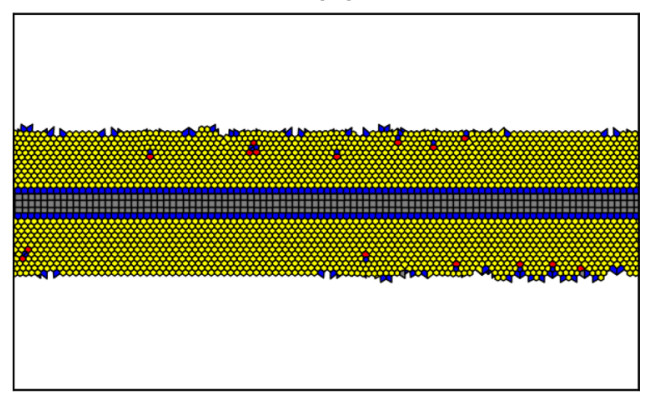

(e)

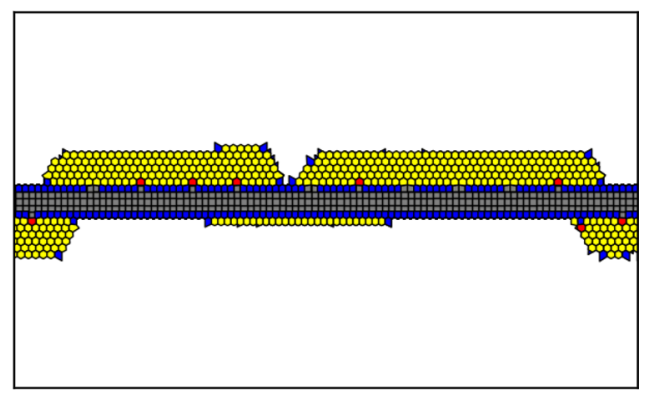

(f)

Fig. 4-Heteroepitaxy in continuous cooling as predicted by the PFC model in the case of high anisotropy of the free energy of the crystal-liquid interface. (Here $\psi_{0}=-0.5$, while the reduced temperature $\varepsilon$ increases linearly in time from 0.4 to 0.75 in $3 \times 10^{5}$ dimensionless time steps, corresponding to cooling from above the melting point, and a strongly anisotropic interface free energy yielding a highly faceted crystal morphology all the time): ( $a$ through $c$ ) Particle density maps. ( $d$ through $f$ ) Analysis in terms of Voronoi polyhedra. (a, d) Layer-by-layer growth (van der Meerwe mechanism); (b, e) layer-by-layer growth followed by island formation (Stranski-Krastanov mechanism); and (c, f) island formation (Volmer-Weber mechanism/heterogeneous nucleation). In panels ( $\mathrm{d}$ through $\mathrm{f}$ ) the colors correspond to different numbers of the neighbors: gray, blue, yellow, and red stand for 4, 5, 6, and 7 neighbors, respectively. From top to bottom $\sigma / \sigma_{0}=1.0,1.19$, and 0.86 . 


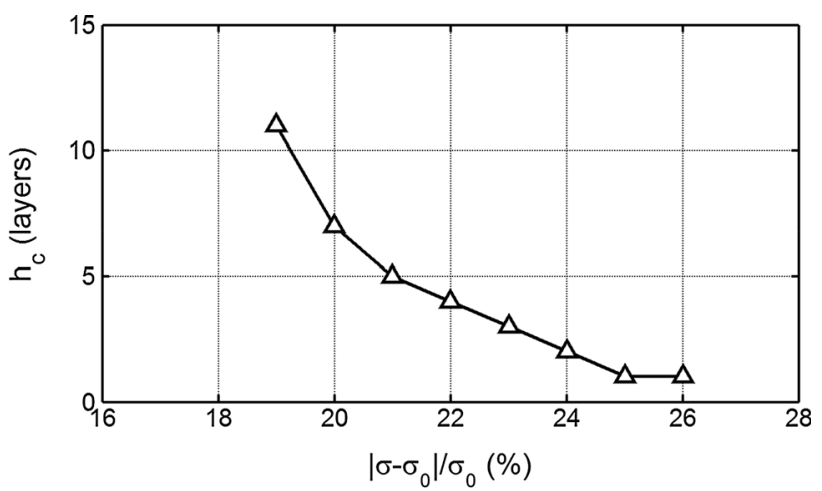

(a)

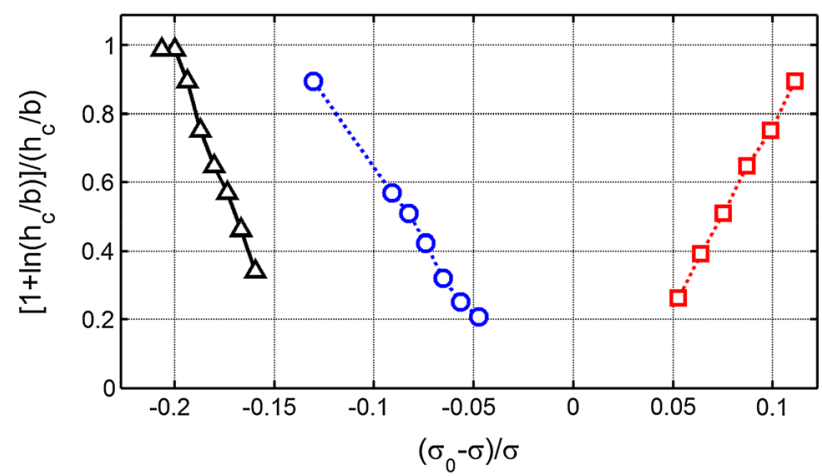

(b)

Fig. 5-Stress release via misfit dislocations in a faceted (high anisotropy) system: (a) Critical thickness of layer-by-layer growth vs, lattice mismatch. (b) The Matthews-Blakeslee plot (triangles). Note that a considerable deviation from linearity is needed to connect these results to the origin. (For comparison the results for low anisotropy (circles and squares) are also shown.).

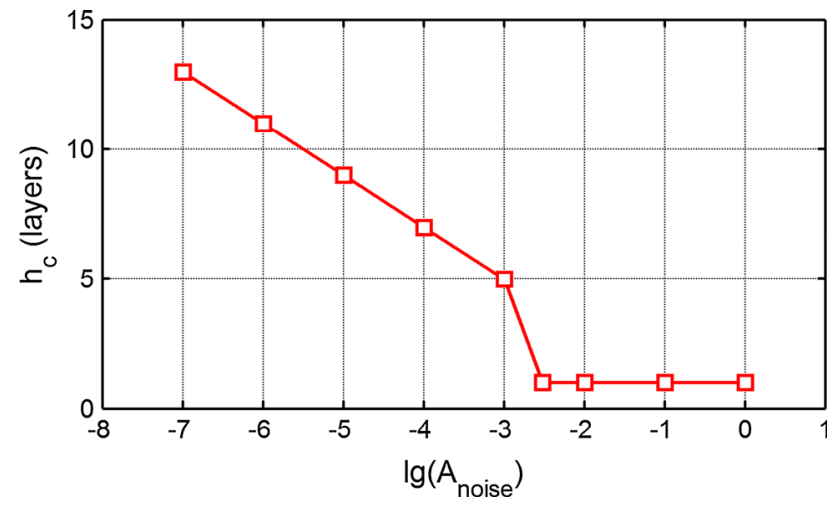

Fig. 6 - Critical thickness $v s$ noise strength.

when the orientation (01-1) is parallel to the wall (Figure $8(\mathrm{~d})$ ), whereas $\theta=90 \mathrm{deg}$ for (11-2) being parallel to the wall (Figure 8(e)).

\section{Nucleation barrier}

The work of formation of the equilibrium clusters fits well to the classical $W(l)=\mathrm{A} l^{2}+\mathrm{B} l$ relationship, where $l$ is the linear size of the nucleus (see Figure 7). ${ }^{[24]}$ The nucleation barrier $\left(W^{*}\right)$ can be defined as the maximum of the fitted formula. Figure 8(f) displays $W^{*}$ obtained so for the two orientations in the $1 / 2<\sigma / \sigma_{0} \leq 2$ regime. Apparently, the $W^{*}$ vs $\sigma / \sigma_{0}$ relationship is nonmonotonic, and has deep minima for the matching lattice constants $\left(\sigma / \sigma_{0}=1\right.$ and $\sqrt{3}$ for the two orientations). Except for extremely large lattice mismatch, nuclei having the direction (01-1) parallel with the wall dominate.

Summarizing, neither the contact angle nor the nucleation barrier are monotonic functions of the lattice mismatch.

\section{B. Complex Substrate Shapes}

The method applied here allows the use of various substrate shapes. Examples of the possibilities are shown in Figure 9, which include sinusoidal and rectangular grooves, saw-tooth shape, and atomic scale steps, and particles of various shape (e.g., square). A detailed analysis of the influence of the surface shape/roughness on crystal nucleation and growth is left for the future. Herein, we concentrate exclusively on rectangular corners and particles.

\section{Rectangular inner corners}

Crystal nucleation inside rectangular corners has been investigated recently in the framework of the PFC model. ${ }^{23,25,47]}$ It has been found that unlike in the classical nucleation theory ${ }^{[1]}$ or the coarse-grained phase-field theory ${ }^{[51]}$ the rectangular corner is not a preferred site for the triangular crystal, as different crystallographic orientations form on the two substrate faces forming the corner, which requires the formation of a grain boundary at the corner, making it energetically unfavorable. The same is true for a substrate with rectangular grooves (Figure 10(a)): note the dislocation chains (blue-red chains) emerging from the internal corners in the Voronoi map (Figure 10(b)).

\section{Crystallization induced by nanoparticles}

Next we recall the results the PFC model predicted recently for the free-growth limited mechanism of particle-induced crystallization for square-shaped nanoparticles in $2 \mathrm{D} .^{[24,25]}$ We note that for such particles the free-growth limited model predicts a critical shape composed by placing half of the homogeneous nucleus on all the four faces of the substrate.

First, we address the case of weak anisotropy (observable, e.g., at a reduced temperature of $\varepsilon=0.25$ ): We approximate nearly perfect wetting (an assumption of the free-growth limited model), via setting $\sigma=\sigma_{0}$. (This is not fully ideal wetting as the substrate and the crystal have different crystal structures.) Two drastically different particle sizes are considered: $L_{\mathrm{s}}=4 \sigma$ and $L_{\mathrm{s}}=32 \sigma$.

In the case of the larger nanoparticle $\left(L_{\mathrm{s}}=32 \sigma\right)$, we found that even outside/above the liquidus line, adsorbed crystal layers form on the surface of the substrate (see Figure 11(a)), which transform into circular "caps" inside the coexistence region (Figure 11(b)). This continues until the diameter of the homogeneous nucleus becomes larger than $L_{\mathrm{s}}$, and free growth sets in (Figure 11(c)). ${ }^{[24]}$ These findings are in a good agreement with the free-growth limited model. 


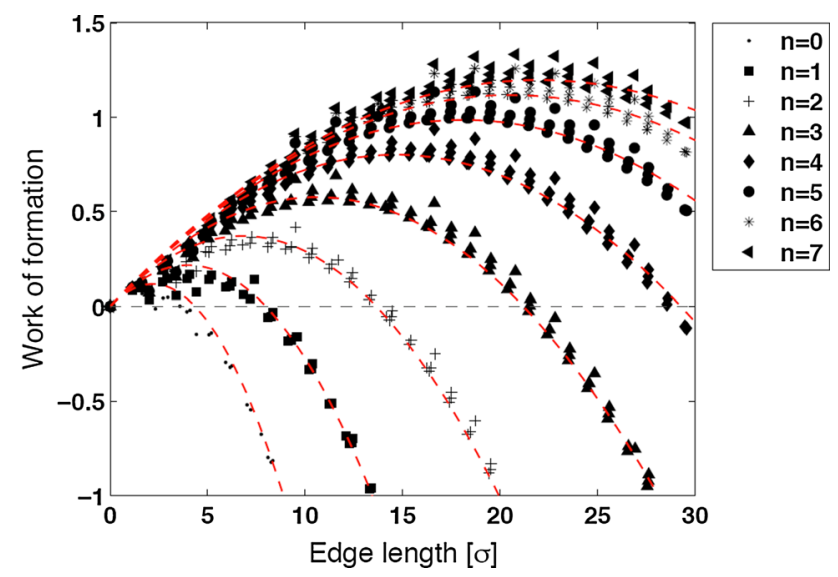

Fig. 7-Dimensionless nucleation barrier for 2D heterogeneous nucleation $v s$ size relationship obtained by solving the ELE for faceted nuclei. (The initial density $\psi_{0}$ decreases with increasing $n$ as follows: $\psi_{0}^{n}=-0.5139+0.002 / 2^{n}$, while $\varepsilon=0.5$ ). The lattice constant of the square-lattice substrate is equal to the interparticle distance of the triangular crystal. Here, the "edge length" is the length of the free side of the crystallites parallel with the substrate (see Figure $8(\mathrm{e})$ ). (Reprinted with permission from Tóth et al. ${ }^{[44]}$ (c) 2010 Institute of Physics.).

In agreement with previous observations for homogeneous nucleation, ${ }^{[52]}$ in the case of the smaller nanoparticle $\left(L_{\mathrm{s}}=4 \sigma\right)$ a faceted crystal shape is observed, and the free growth takes place at a supersaturation where the critical size is a monatomic cluster that is significantly smaller than $L_{\mathrm{s}}$ (see Figures 11(d) through (f)).

In turn, at $\varepsilon=0.5$, the crystal habit is faceted for all sizes (Figures 11(g) through (i)), and free growth takes place at a supersaturation, where the critical fluctuation is the nearest neighbor cluster that is far smaller than $L_{\mathrm{s}}=32 \sigma$.

These findings indicate that the original free-growth limited model of particle-induced freezing works so far as the foreign particles are sufficiently large, and the crystal-liquid interfacial free energy has a weak anisotropy.

The validity of the free-growth limited model of particle-induced solidification has also been investigated in $3 \mathrm{D} \cdot{ }^{[24,25]}$ Cube-shaped nanoparticles of simple cubic (sc) structure have been considered, with a lattice constant, $a_{\mathrm{s}}$, which is the same as the lattice constant of the bcc structure. Particle-induced freezing has been studied in the stability domain of the bcc structure. ${ }^{[24]}$ The PFC simulations are in a qualitative agreement with the free-growth limited model. Remarkably, the critical shape preceding free growth depends on the size of the foreign particle. The large particle limit for the critical shape from a surface solver by Reavley and Greer $^{[21]}$ (Figure 12(a)) is compared with morphologies predicted by PFC simulations for $L_{\mathrm{s}}=32 a_{\mathrm{s}}$ and $L_{\mathrm{s}}=$ $16 a_{\mathrm{s}}$ (see Figures 12(b) and (c)). Apparently, the critical shape is size dependent: While the first is a continuously curving surface with eight corners, the $L_{\mathrm{s}}=32 a_{\mathrm{s}}$ cluster has spherical caps on the (100) faces, whereas in the case of the $L_{\mathrm{s}}=16 a_{\mathrm{s}}$ pyramids form on the (100) faces.

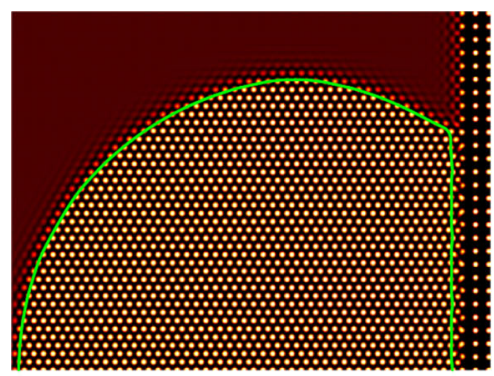

(a)

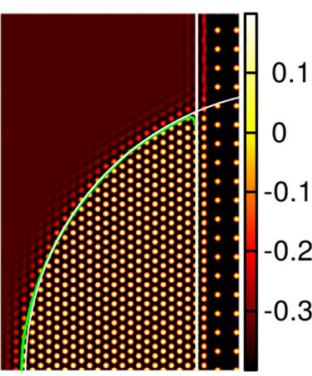

(b)

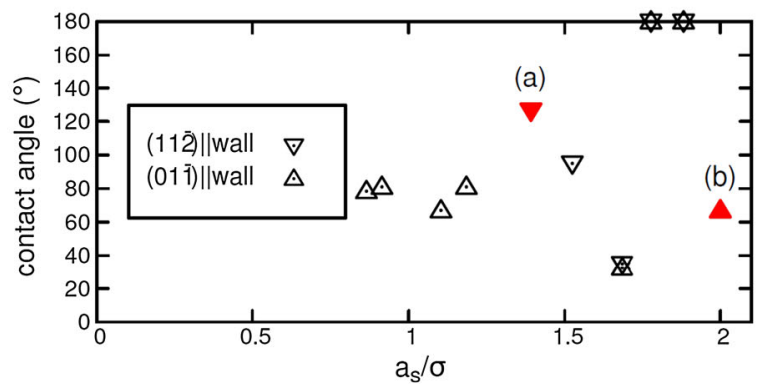

(c)

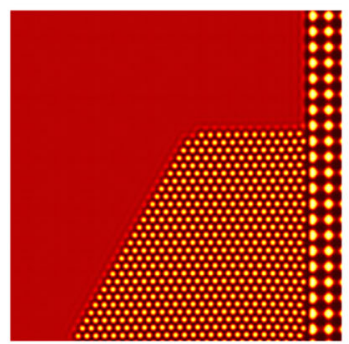

(d)

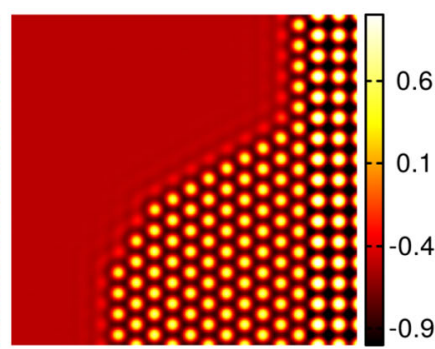

(e)

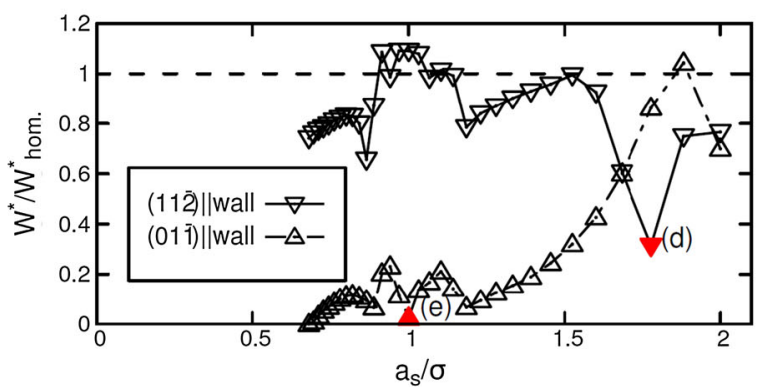

(f)

Fig. 8-Heterogeneous nucleation on a flat wall in 2D from solving ELE for square-lattice substrate. ${ }^{[24]}(a, b)$ Typical nonfaceted nuclei seen at small anisotropies. Here $\sigma / \sigma_{0}=1.49$ and 2.0, respectively, while the orientations are (11-2) and (01-1) parallel with the wall. The intersection of the circular and linear fits (white lines) to the contour line (green) defines the contact angle. (c) Contact angle vs $\sigma /$ $\sigma_{0}$ for small anisotropy. The full triangles stand for cases shown in panels (a) and (b). $(d, e)$ Faceted nuclei obtained far from the critical point, at $\sigma / \sigma_{0}=\sqrt{3}$ and 1.0. Respective orientations: (11-2) and (011) parallel with the wall. $(f)$ Work of formation of faceted nuclei normalized by the value for homogeneous nucleation $\left(W^{*}=W^{*}\right.$ hom $)$ $v s \sigma / \sigma_{0}$. The full triangles stand for cases shown in panels (d) and (f). (Reprinted with permission from Tóth et al. ${ }^{[2]}$ (C) 2012 American Physical Society.).

Finally, it is worth noting that varying the lattice constant of an fcc substrate with a rectangular pit on its surface initiates bcc and fcc solidification for matching 

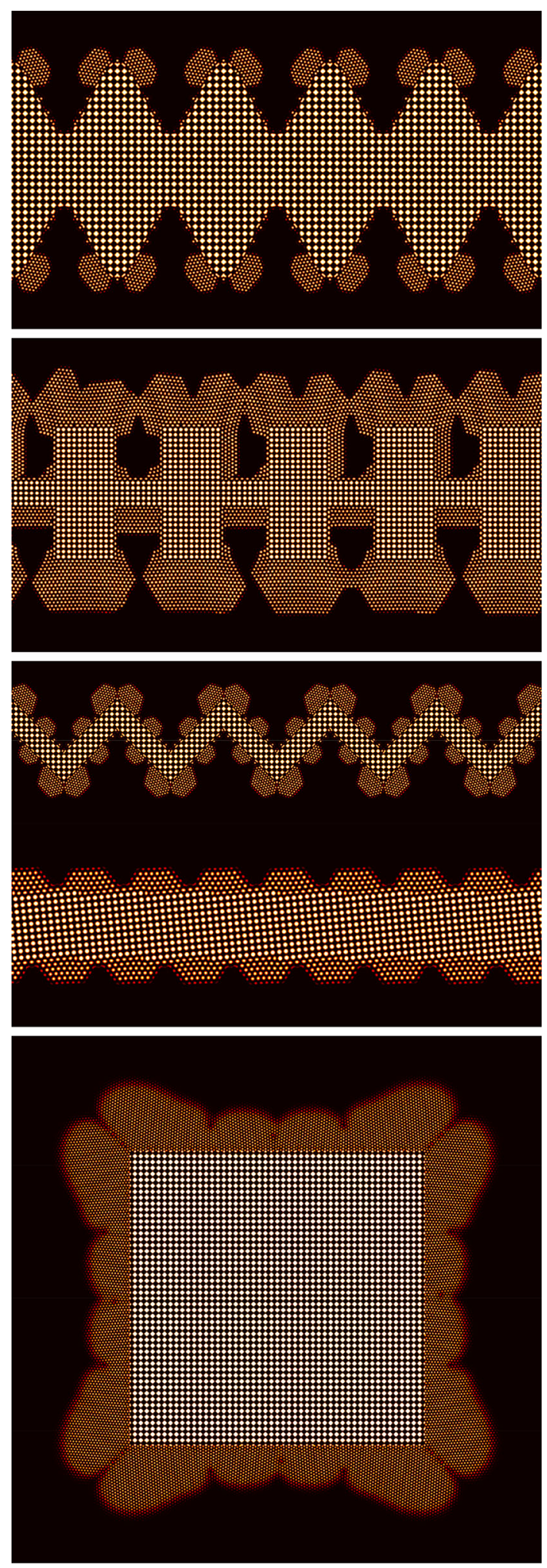

Fig. 9-Solidification on crystalline substrates of various shapes. Areas of lighter color indicate the substrate.

lattice constant, ${ }^{[24]}$ (a phenomenon analogous to the one observed for a simple cubic substrate $\left.{ }^{[53]}\right)$, whereas for extreme mismatch an amorphous layer forms, ${ }^{[24]}$ which in turn initiates the nucleation of the bcc structure
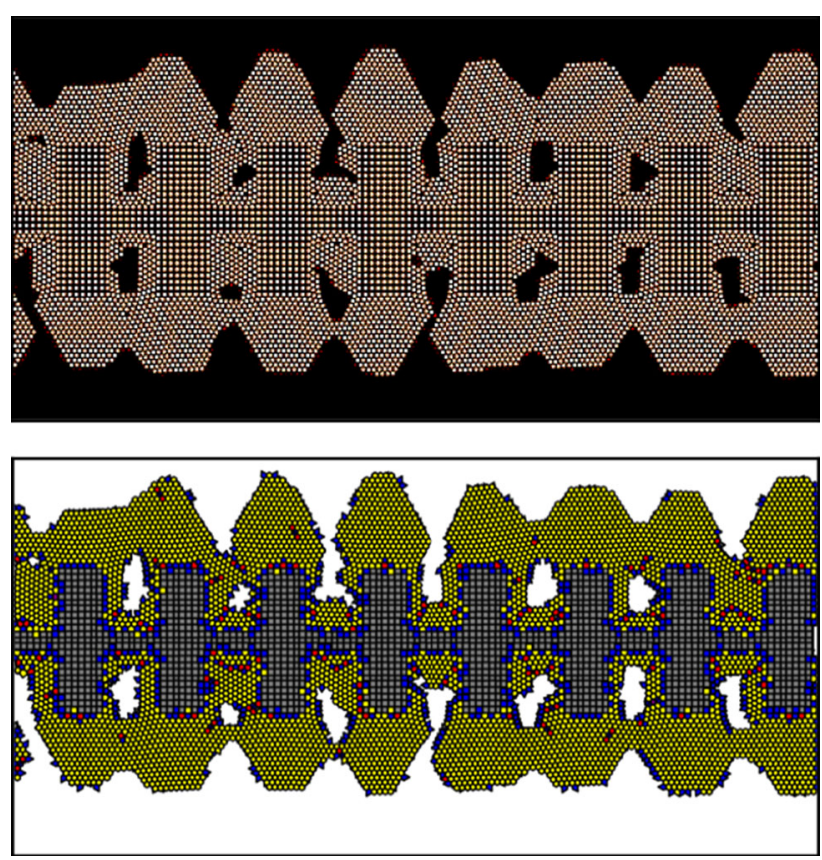

Fig. 10 - Crystallization in rectangular grooves during continuous cooling for large anisotropy. Upper panel: density distribution (for the sake of better visibility of the individual particles the contrast has been increased). Lower panel: Voronoi polyhedral analysis. Note the dislocation chains (consisting 5 and 7 coordinated particles) emerging from the inner corners.

(Figure 13), in analogy to the amorphous precursormediated crystallization. ${ }^{[38]}$

\section{SUMMARY}

Recent developments in PFC modeling of heteroepitaxy and heterogeneous crystal nucleation on flat and modulated surfaces and nanocrystals have been briefly reviewed. It has been found that

1. in heteroepitaxy of hexagonal crystal of small anisotropy (close to the critical point) on a square-lattice substrate the misfit dislocations nucleate in the cusps of the solid-liquid interface formed owing to the Asaro-Tiller-Grinfeld instability, and then travel to the surface of the substrate, while the critical thickness for which the strained crystal is stable follows the Matthews-Blakeslee scaling;

2. in contrast, for heteroepitaxy of a crystalline phase with large anisotropy (which also means larger distance from the critical point), the amplitude of the surface undulations via the Asaro-Tiller-Grinfeld instability is smaller, and a large deviation from the Matthews-Blakeslee scaling is observed, presumably because of the nonlinear elasticity prevailing in this regime;

3. the lattice mismatch between a square-lattice substrate and the trigonal crystal influences nonmonotonically such properties as the contact angle, the thickness of the crystalline layer adsorbed on the substrate, and the height of the thermodynamic barrier for heterogeneous nucleation; 

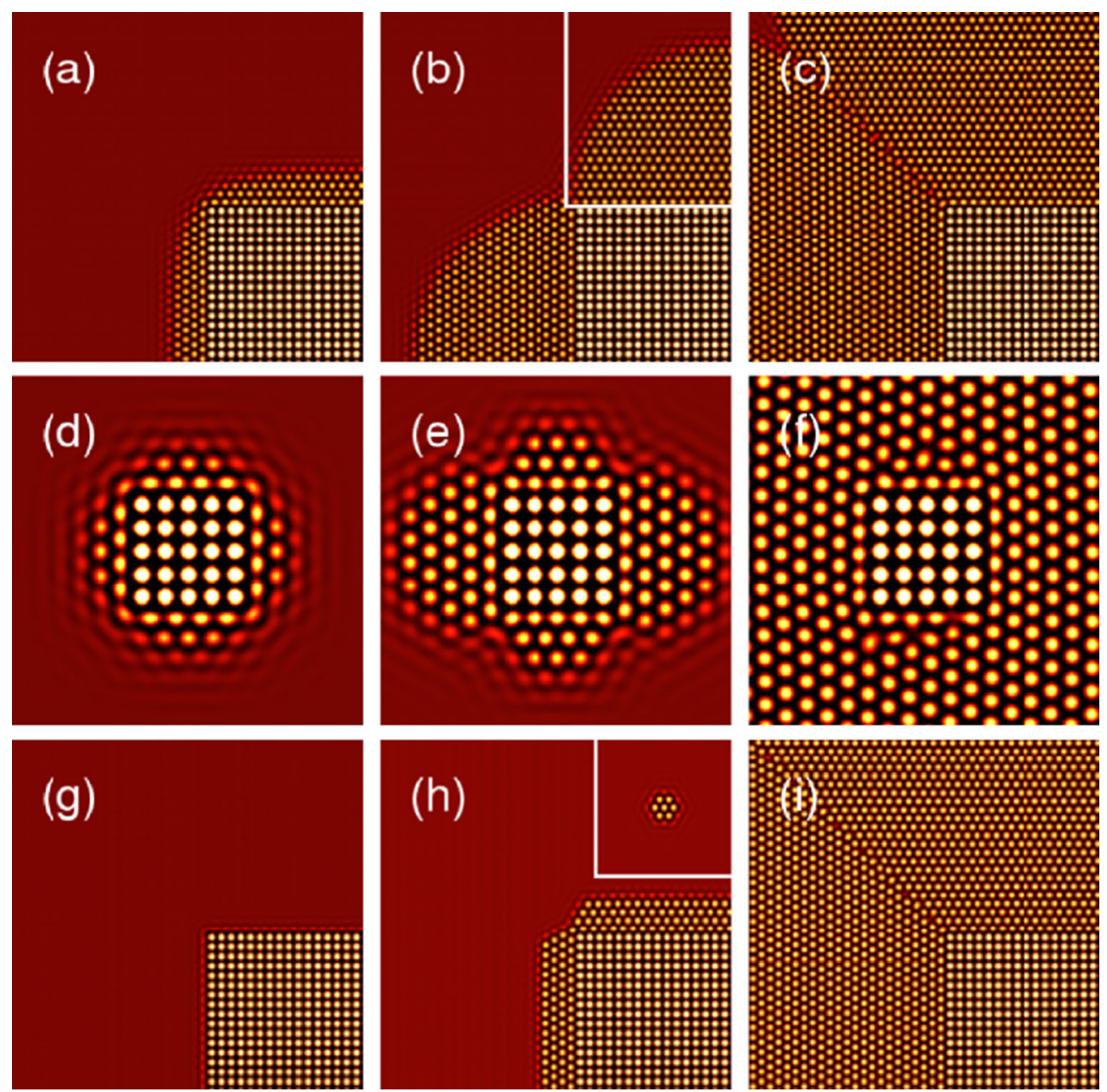

Fig. 11-Free-growth-limited mode of particle-induced freezing in 2D on square-shaped nanoparticles of square-lattice structure in the PFC model, as predicted by the ELE. The liquid density, the reduced temperature, and the particle size are as follows: $(a$ through $c) \varepsilon=0.25$ and $L_{\mathrm{s}}$ $=32 \sigma ; \psi_{0}=-0.3418,-0.3405$, and -0.3404 . ( $d$ through $\left.f\right) \varepsilon=0.25$, and $L_{\mathrm{s}}=4 \sigma$; whereas $\psi_{0}=-0.3426,-0.3363$, and -0.3359 . ( $g$ through i) $\varepsilon=0.5$ and $L_{\mathrm{s}}=32 \sigma ; \psi_{0}=-0.5190,-0.4939$, and -0.4929 . In all cases $\sigma_{0} / \sigma=1$. The insets display the homogeneous nuclei corresponding to the supersaturation at free growth. (Reprinted with permission from Tóth et al. ${ }^{[2]}$ (C) 2012 American Physical Society.).

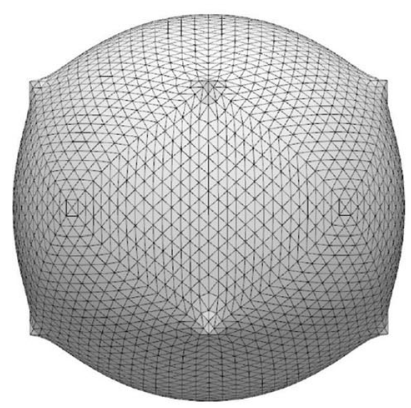

(a)

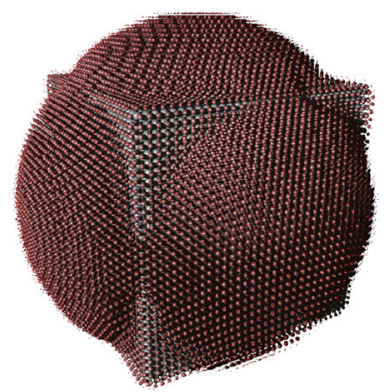

(b)

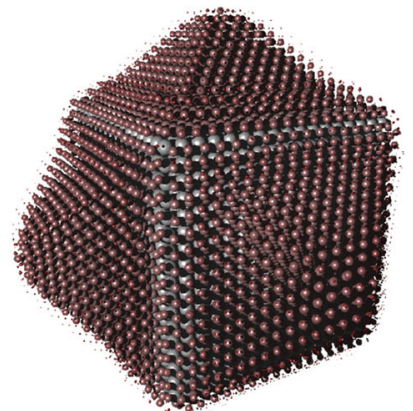

(c)

Fig. 12-Stable shapes preceding free growth in the free-growth-limited mode of particle-induced crystallization as a function of the linear size of the cubic particles. (a) Theoretical shape for infinite size, ${ }^{[21]}$ and PFC predictions for $(b) L_{\mathrm{s}}=32 a_{\mathrm{s}}$ and $(c) L_{\mathrm{s}}=16 a_{\mathrm{s}}$. Note that with decreasing size a faceted shape develops as reported for homogeneous nucleation by Backofen and Voigt. ${ }^{[52]}$ 

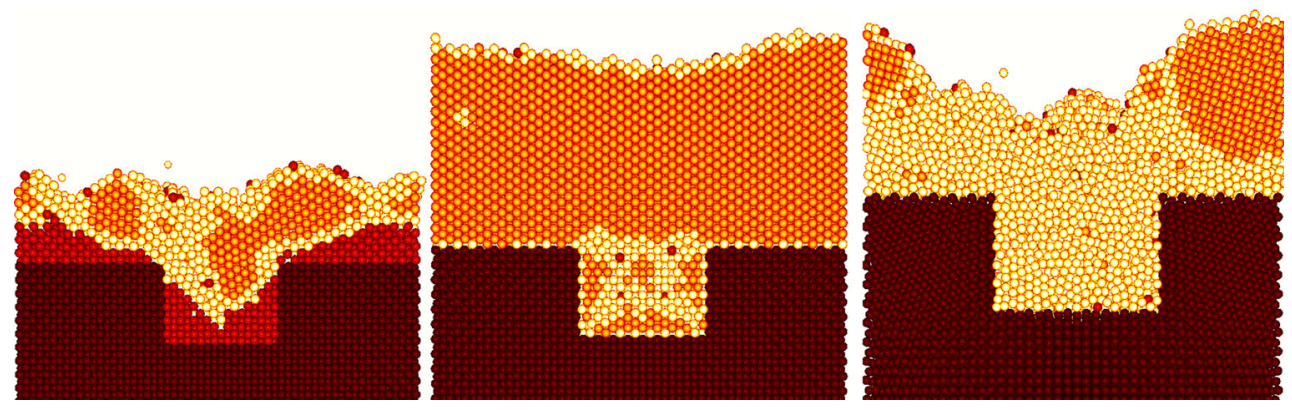

Fig. 13 - Crystallization on fcc substrate with a rectangular nanoscale pit (equation of motion in 3D). ${ }^{[24]}$ Spheres drawn around density peaks larger than a threshold are shown. Bond order parameters $q_{4}$ and $q_{6}$ have been used for the structural analysis. Hues changing from dark to light stand for the substrate, and the fcc, bcc, and amorphous structures, respectively. ( $\varepsilon=0.16$ and $\left.\psi_{0}=-0.25\right)$ From left to right the ratio of the lattice constants are $a_{\mathrm{s}} / a_{\mathrm{fcc}}=1.0,1.098$, and 1.42. Cross-sectional views are displayed. (Reprinted with permission from Tóth $e t$ al. ${ }^{[24]} \odot$ 2012 American Physical Society.).

4. the highly successful free-growth limited model of particle-initiated crystallization by Greer and coworkers $^{[19-21]}$ appears to be valid for larger nanoparticles (e.g., $\left.L_{\mathrm{s}}=32 \sigma\right)$ and small anisotropy of the solid-liquid interface free energy, whereas for small nanoparticles (e.g., $L_{\mathrm{s}}=4 \sigma$ ) or for faceted crystals, the critical supersaturation, beyond which free growth takes place, substantially differs from the prediction of analytic theory;

5. a large lattice mismatch between the crystal and the substrate may lead to the formation of an amorphous surface layer, which then might assist the formation of the crystalline phase; a phenomenon that can be considered as a heterogeneous analog of the amorphous precursor-mediated homogeneous crystal nucleation.

\section{ACKNOWLEDGMENTS}

This work includes techniques developed in the framework of the EU FP7 Collaborative Project "EXOMET" (Contract No. NMP-LA-2012-280421, co-funded by ESA), and by the ESA MAP/PECS projects MAGNEPHAS III, PARSEC, and GRADECET.

\section{REFERENCES}

1. K.F. Kelton and L. A. Greer: Nucleation in Condensed Matter. Pergamon Materials Series, vol. 15 (Elsevier, Amsterdam, 2010).

2. B.A. Grzybowski, K.J.M. Bishop, C.J. Campbell, M. Fialkowski, and S.K. Smoukov: Soft Matter, 2005, vol. 1, pp. 114-28 and references therein.

3. J. Aizenberg, A.J. Black, and G.M. Whitesides: Nature, 1999, vol. 398, pp. 495-98.

4. C.X. Cui, Y.H. Chen, P. Jin, B. Xu, Y.Y. Ren, C. Zhao, and Z.G. Wang: Physica E, 2006, vol. 31, pp. 43-47.

5. K.-H. Chen, C.-Y. Chien, W.-T. Lai, T. George, A. Scherer, and P.-W. Li: Cryst. Growth Des., 2011, vol. 11, pp. 3222-26.

6. A.J.M. Mackus, M.A. Verheijen, N. Leick, A.A. Bol, and W.M.M. Kessel: Chem. Mater., 2013, vol. 25, pp. 1905-11.

7. G.I. Tóth, J. R. Morris and L. Gránásy, Phys. Rev. Lett., 2011, vol. 106, art. no. 045701.

8. W. Cheng, N. Park, M.T. Walter, M. Hartman, and D. Luo: Nat Nanotechnol., 2008, vol. 3, pp. 682-690.
9. S. Auer and D. Frenkel: Phys. Rev. Lett., 2003, vol. 91, art. no. 015703 .

10. D. Winter, P. Virnau, and K. Binder: Phys. Rev. Lett., 2009, vol. 103 , art. no. 225703.

11. M. Heni and H. Löwen: J. Phys.: Condens. Matter, 2001, vol. 13, pp. 4675-96.

12. A. Esztermann and H. Löwen: J. Phys.: Condens. Matter, 2005, vol. 17, pp. S429-41.

13. S. Toxvaerd: J. Chem. Phys., 2002, vol. 117, pp. 10303-10.

14. E.B. Webb III, G.S. Grest and D.R. Heine: Phys. Rev. Lett., 2003 , vol. 91 , art. no. 236102

15. L. Gránásy, T. Pusztai, D. Saylor, and J.A. Warren: Phys. Rev. Lett., 2007, vol. 98, art. no. 035703.

16. J.A. Warren, T. Pusztai, L. Környei, and L. Gránásy: Phys. Rev. $B, 2009$, vol. 79 , art. no. 014204 .

17. S. van Teeffelen, C.N. Likos, and H. Löwen: Phys. Rev. Let., 2008, vol. 100 , art. no. 108302 .

18. T. Neuhaus, M. Marechal, M. Schmiedeberg, and H. Löwen: Phys. Rev. Lett., 2013, vol. 110, art. no. 118301.

19. A.L. Greer, A.M. Brunn, A. Tronche, P.V. Evans, and D.J. Bristow: Acta Mater., 2000, vol. 48, pp. 2823-35.

20. T.E. Quested and A.L. Greer: Acta Mater., 2005, vol. 53, pp. 2683-92.

21. S.A. Reavley and A.L. Greer: Philos. Mag., 2008, vol. 88, pp. 56179.

22. K.R. Elder, M. Katakowski, M. Haataja and M. Grant: Phys. Rev. Lett., 2002, vol. 88, art. no. 245701.

23. H. Emmerich, H. Löwen, R. Wittkowski, T. Gruhn, G.I. Tóth, G. Tegze, and L. Gránásy: Adv. Phys., 2012, vol. 61, pp. 665-743 and references therein.

24. G.I. Tóth, G. Tegze, T. Pusztai, and L. Gránásy: Phys. Rev. Lett., 2012, vol. 108, art. no. 025502

25. L. Gránásy, F. Podmaniczky, G.I. Tóth, G. Tegze, and T. Pusztai: Chem. Soc. Rev., 2014, vol. 43, pp. 2159-73.

26. Z. Fan: Proc. J. Hunt Int. Symposium, Z. Fan and I.C. Stone, eds., Brunel University Press, Uxbridge, 2001, pp 29-44.

27. Z. Fan: Metall. Mater. Trans. A, 2013, vol. 44A, pp. 1409-18.

28. O. Galkin and P. Vekilov: Proc. Natl. Acad. Sci. USA, 2000, vol. 97, pp. 6277-81.

29. P.G. Vekilov: Cryst. Growth Des., 2004, vol. 4, pp. 671-85.

30. P.R. TenWolde and D. Frenkel: Science, 1997, vol. 277, pp. 197578.

31. V. Talanquer and D.W. Oxtoby: J. Chem. Phys., 1998, vol. 109, pp. $223-27$.

32. G.I. Tóth and L. Gránásy, J. Chem. Phys., 2007, vol. 127, art. no. 074710 .

33. T. Kawasaki and H. Tanaka: Proc. Natl. Acad. Sci. USA, 2010, vol. 107, pp. 14036-41.

34. T.H. Zhang and X.Y. Liu: J. Am. Chem. Soc., 2007, vol. 129, pp. $13520-26$.

35. H.J. Schöpe, G. Bryant, and W. van Megen: Phys. Rev. Lett., 2006, vol. 96, art. no. 175701.

36. J.F. Lutsko and G. Nicolis, Phys. Rev. Lett., 2006, vol. 96, art. no. 046102 . 
37. T. Schilling, H.J. Schöpe, M. Oettel, G. Opletal, and I. Snook: Phys. Rev. Lett., 2010, vol. 105, art. no. 025701.

38. G.I. Tóth, T. Pusztai, G. Tegze, G. Tóth, and L. Gránásy: Phys. Rev. Lett., 2011, vol. 107, art. no. 175702.

39. K.R. Elder, N. Provatas, J. Berry, P. Stefanovic, and M. Grant: Phys. Rev. B, 2007, vol. 75, art. no. 064107.

40. S. van Teeffelen, R. Backofen, A. Voigt, and H. Löwen: Phys. Rev. E, 2009, vol. 79, art. no. 051404 .

41. U.M.B. Marconi and P. Tarazona: J. Chem. Phys., 1999, vol. 110, pp. 8032-44.

42. H. Löwen: J. Phys.: Condens. Matter, 2003, vol. 15, pp. V1-V3.

43. A.J. Archer and M. Rauscher: J. Phys. A: Math. Gen., 2004, vol. 37, pp. 9325-33.

44. G.I. Tóth, G. Tegze, T. Pusztai, G. Tóth, and L. Gránásy: $J$. Phys.: Condens. Matter, 2010, vol. 22, art. no. 364101.
45. G. Tegze, G. Bansel, G.I. Tóth, T. Pusztai, Z. Fan, and L. Gránásy: J. Comput. Phys., 2009, vol. 228, pp. 1612-23.

46. R. Backofen and A. Voigt: J. Phys.: Condens. Matter, 2009, vol. 21 , art. no. 464109 .

47. L. Gránásy, G. Tegze, G.I. Tóth, and T. Pusztai: Philos. Mag., 2011, vol. 91, pp. 123-49.

48. J.W. Matthews and A.E. Blakeslee: J. Cryst. Growth, 1974, vol. 27, pp. $118-25$.

49. R.J. Asaro and W.A. Tiller: Metall. Trans., 1972, vol. 3, pp. 1789-96.

50. K.R. Elder and M. Grant: Phys. Rev. E, 2004, vol. 70, art. no. 051605.

51. M. Castro: Phys. Rev. B, 2003, vol. 67, art. no. 035412.

52. R. Backofen and A. Voigt: J. Phys.: Condens. Matter., 2010, vol. 22 , art. no. 364104.

53. G. Tegze, L. Gránásy, G.I. Tóth, F. Podmaniczky, A. Jaatinen, T. Ala-Nissila, and T. Pusztai: Phys. Rev. Lett., 2009, vol. 103, art. no. 035702 . 